\title{
Trend of salvage treatment in diffuse large $B$ cell lymphoma in the outpatient chemotherapy era
}

\author{
AYA NAKAYA, SHINYA FUJITA, ATSUSHI SATAKE, TAKAHISA NAKANISHI, \\ YOSHIKO AZUMA, YUKIE TSUBOKURA, RYO SAITO, AKIKO KONISHI, MASAAKI HOTTA, \\ HIDEAKI YOSHIMURA, KAZUYOSHI ISHII, TOMOKI ITO and SHOSAKU NOMURA
}

First Department of Internal Medicine, Kansai Medical University, Hirakata, Osaka 573-1010, Japan

Received April 24, 2019; Accepted August 20, 2019

DOI: $10.3892 / \mathrm{mco} .2019 .1930$

\begin{abstract}
Patients with diffuse large B cell lymphoma (DLBCL) who have failed to achieve complete remission with first-line therapy can subsequently receive salvage therapy. However, there is no definite consensus on the use of salvage therapy, and little information on the optimal treatment regimen. The present study retrospectively analyzed data from 131 patients diagnosed with DLBCL between April 2002 and November 2017 who relapsed and received salvage therapy. Primary treatment included R-CHOP or R-CHOP-like regimens. The most common salvage regimen was R-DeVIC (42\%), followed by R-ESHAP (23\%), other aggressive regimens (12\%) and palliative therapy (23\%). The median overall survival (OS) was 45.7 months for R-DeVIC, 41.8 months for palliative therapy, 29.4 months for R-ESHAP, and 28.5 months for aggressive regimens $(\mathrm{P}=0.937)$. A total of 25 patients underwent autologous stem cell transplantation (ASCT), and the OS was 75.6 months for these patients compared with 33.5 months (range, 25.6-45.6 months) for patients who did not undergo ASCT $(\mathrm{P}=0.033)$. Following the establishment of an outpatient chemotherapy unit in 2014, R-DeVIC use became more common, increasing from $37 \%$ prior to 2014 to $46 \%$ after 2014, whereas R-ESHAP use decreased (31 to $17 \%$ ). The present study did not identify the optimal salvage regimen for patients with DLBCL. However, salvage ASCT improved the outcome, and regimens administered via peripheral veins were demonstrated to be more common in outpatient chemotherapy settings.
\end{abstract}

\section{Introduction}

In patients with diffuse large B cell lymphoma (DLBCL), rituximab-based chemotherapy regimens can achieve superior long-term progression-free survival (PFS) and overall

Correspondence to: Dr Aya Nakaya, First Department of Internal Medicine, Kansai Medical University, 2-5-1 Shin-machi, Hirakata, Osaka 573-1010, Japan

E-mail: nakaya1016@yahoo.co.jp

Key words: salvage, diffuse large B cell lymphoma, outpatient chemotherapy, R-DeVIC, R-ESHAP survival (OS) rates relative to regimens that do not contain rituximab (1). However, even in the rituximab era, approximately $10-15 \%$ of patients with DLBCL treated with rituximab plus cyclophosphamide, doxorubicin, vincristine, and prednisone (R-CHOP) fail to achieve complete remission (CR) $(2,3)$. Such patients can often be treated with salvage chemotherapy regimens and subsequent autogenous stem cell transplantation (ASCT) $(4,5)$. To date, there has been no definite consensus on salvage therapy in this patient population and little information on the ideal treatment regimen, with data from randomized trials of salvage therapy failing to reveal significant differences between regimens (5). Two randomized clinical studies have compared treatment regimens prior to ASCT. In the Collaborative Trial in Relapsed Aggressive Lymphoma (CORAL) study, R-ICE (rituximab, ifosfamide, carboplatin, etoposide) was compared with R-DHAP (rituximab, cytosine arabinoside, cisplatin, dexamethasone), followed by ASCT with or without rituximab maintenance, and no difference was identified between the regimens (4). In the LY02 study, R-DHAP was compared with R-GDP (rituximab, gemcitabine, dexamethasone, cisplatin) before ASCT. Although no difference in OS was reported, R-GDP was associated with fewer AEs, improved quality of life (QOL), and less-frequent hospitalization (5). In a recent patient-level analysis of outcomes of refractory DLBCL from two large randomized trials and two academic databases (SCHOLAR-1) (6), an objective response rate of 26\% (CR 7\%) to the next line of therapy was demonstrated, with a median OS of 6.3 months. This analysis also showed that a poor outcome was associated with relapsed and refractory DLBCL. The results from these previous studies indicate a clear need for novel therapies to improve outcomes in this patient population.

We therefore sought to determine the safest and most effective regimen in the clinical setting. Furthermore, with current treatment trends, we determined which regimens are changing from administration in an inpatient to an outpatient setting.

\section{Patients and methods}

Patients. This retrospective analysis was conducted at Kansai Medical University Hospital and Kansai Medical University Medical Center. Among 530 patients diagnosed with DLBCL from April 2002 to November 2017, 131 relapsed and refractory patients who received salvage therapy were enrolled in this 
study. Primary treatment included R-CHOP or R-CHOP-like regimens. Tumor responses were assessed according to the classification of the International Workshop to Standardize Response Criteria for Non-Hodgkin's Lymphoma 1999. Relapse was defined as emerging new sites or enlarging sites and refractory disease was defined as progressive/stable disease during first-line treatment. Performance status was evaluated by an Eastern Cooperative Oncology Group (ECOG) performance status score. To predict prognosis, we used both the international prognostic index (IPI) and the National Comprehensive Cancer Network-International Prognostic Index (NCCN-IPI). Although IPI was the most powerful prognostic scale before the advent of rituximab, its power has reduced (7). NCCN-IPI has been reported more accurate than older IPI in rituximab era (8). However, NCCN-IPI has not been evaluated enough in Japan, thus, we used both scales.

Statistical analysis. Progression-free survival 2 (PFS2) was defined as the period from the start of initial treatment to the second exacerbation or death after second-line treatment. OS was calculated as the time from diagnosis until the time of death or the last clinical follow-up.

Survival curves were generated using the Kaplan-Meier method, and differences were evaluated using the log-rank test. Multivariate Cox-proportional hazards models were used to determine whether baseline characteristics were associated with PFS2 and OS. All statistical tests were two-sided, statistical significance was defined as $\mathrm{P}<0.05$, and $95 \%$ confidence intervals (CIs) were calculated. All statistical analyses were performed using EZR (Saitama Medical Center, Jichi Medical University, Saitama, Japan), a graphical user interface for $\mathrm{R}$ version 2.13.0 (The R Foundation). Specifically, EZR is a modified version of $\mathrm{R}$ Commander (version 1.6-3) that adds statistical functions frequently used in biostatistics (9). Toxicity was evaluated according to the Common Terminology Criteria for Adverse Events (AEs) (CTCAE 4.0, U.S. DEPARTMENT OF HEALTH AND HUMAN SERVICES, National Institutes of Health, National Cancer Institute, [http://ctep.cancer.gov/protocolDevelopment/electronic_applications/ctc.htm\#ctc_40]). This study was conducted in accordance with the ethical principles of the Declaration of Helsinki, and was approved by the institutional review board of Kansai Medical University.

\section{Results}

Patient characteristics. Clinical characteristics of the 131 enrolled patients (median age, 68 years; age range, 35-87 years; $54 \%$ male) are shown in Table I. PS over 2 was $7 \%$. Stage I was $11 \%$, Stage II was $16 \%$, Stage III was $18 \%$, and Stage IV was $55 \%$. Using the IPI, $22 \%$ of patients were classified as being at low risk, $18 \%$ as low-intermediate (LI) risk, $22 \%$ as high-intermediate (HI) risk, and $38 \%$ as high risk. In comparison, under the National Comprehensive Cancer Network (NCCN)-IPI, 7\% of patients were classified as being at low risk, $40 \%$ as LI, $50 \%$ as $\mathrm{HI}$, and $3 \%$ as high risk. The median follow-up period was 33.6 months (range, 1.9-155.9 months).

Salvage regimens. The most common salvage regimen was R-DeVIC (rituximab, etoposide, dexamethasone, ifosfamide,
Table I. Patient characteristics.

\begin{tabular}{lc}
\hline Characteristics & Number $(\%)$ \\
\hline Number of patients & 131 \\
Median age, years (range) & $68(35-87)$ \\
Male & $71(54)$ \\
PS $\geq 2$ & $9(7)$ \\
Stage & \\
I & $14(11)$ \\
II & $21(16)$ \\
III & $24(18)$ \\
IV & $72(55)$ \\
IPI & \\
Low & $29(22)$ \\
Low-int & $23(18)$ \\
High-int & $29(22)$ \\
High & $50(38)$ \\
NCCN-IPI & \\
Low & $10(7)$ \\
Low-int & $52(40)$ \\
High-int & $65(50)$ \\
High & $4(3)$ \\
\end{tabular}

PS, performance status; int, intermediate; IPI, International Prognosis Index; NCCN-IPI, The National Comprehensive Cancer NetworkInternational Prognosis Index.

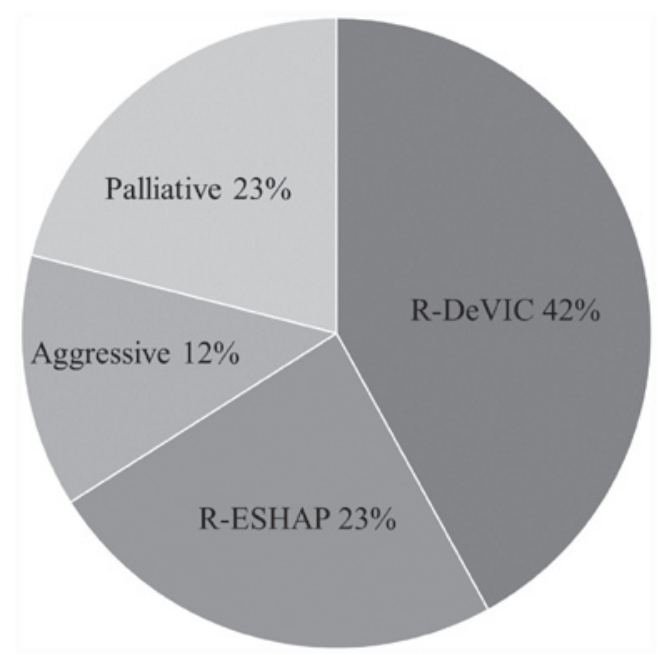

Figure 1. Percentages of salvage therapies. The aggressive regimen was applied in $12 \%$ of cases, and included R-CHASE $(n=5)$, rituximab plus methotrexate-based $(n=5)$, R-CHOP like $(n=3), \operatorname{R-GDP}(n=2)$ and R-EPOCH $(n=1)$. Palliative therapy included radiation only, rituximab monotherapy, oral etoposide and oral prednisolone. R-CHASE, rituximab, cyclophosphamide, cytosine arabinoside, etoposide, dexamethasone; R-GDP, rituximab, gemcitabine, cisplatin, dexamethasone; R-EPOCH, rituximab, etoposide, vincristine, doxorubicin, cyclophosphamide, prednisolone; R-DeVIC, rituximab, etoposide, dexamethasone, ifosfamide, carboplatin; R-ESHAP, rituximab, etoposide, solumedrol, cytarabine, cisplatin.

carboplatin) (42\%), followed by R-ESHAP (rituximab, etoposide, solumedrol, cytarabine, cisplatin) (23\%) (Fig. 1). Other aggressive regimens were administered to $12 \%$ of patients, and 

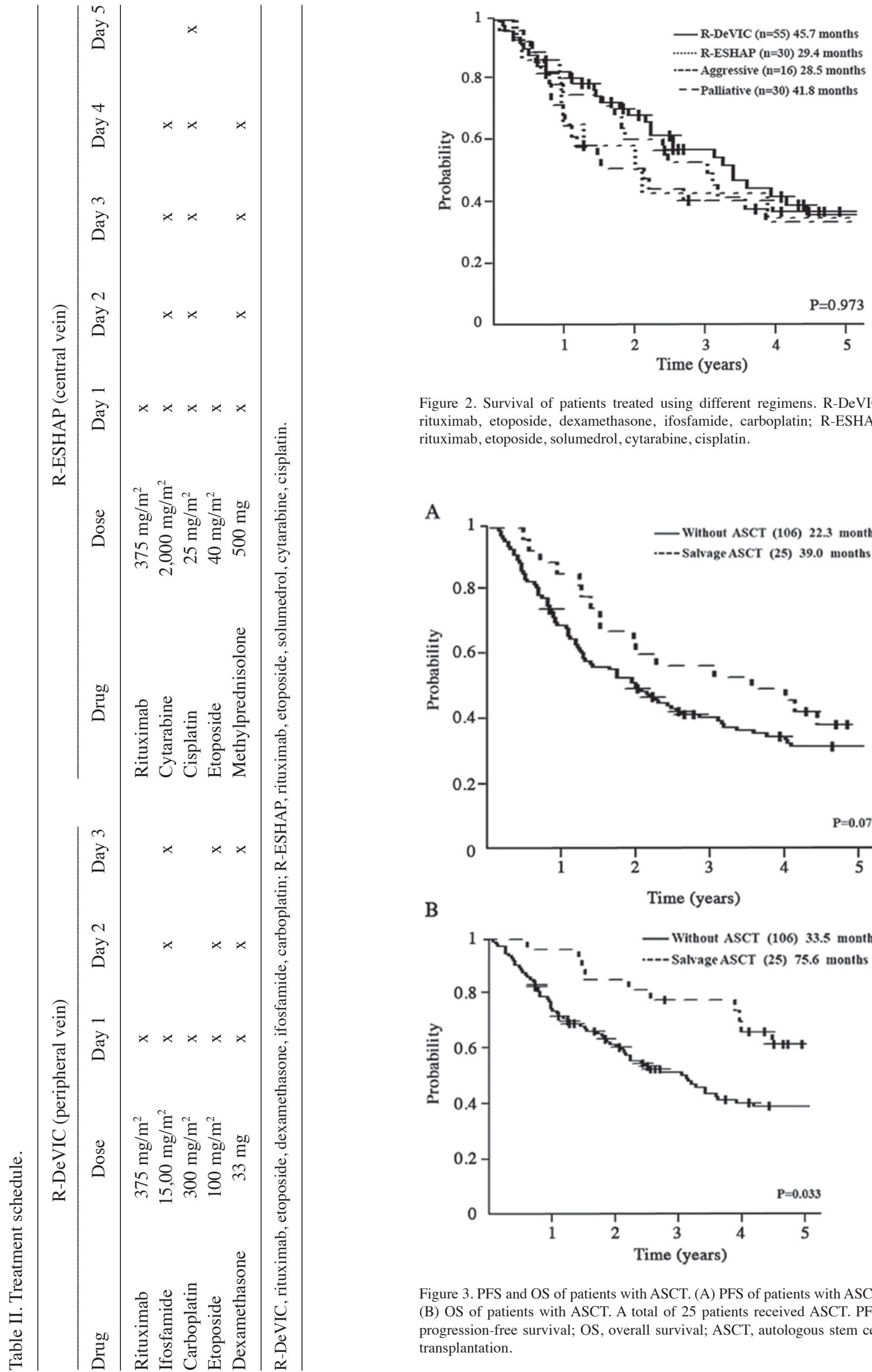

Figure 2. Survival of patients treated using different regimens. R-DeVIC, rituximab, etoposide, dexamethasone, ifosfamide, carboplatin; R-ESHAP, rituximab, etoposide, solumedrol, cytarabine, cisplatin.
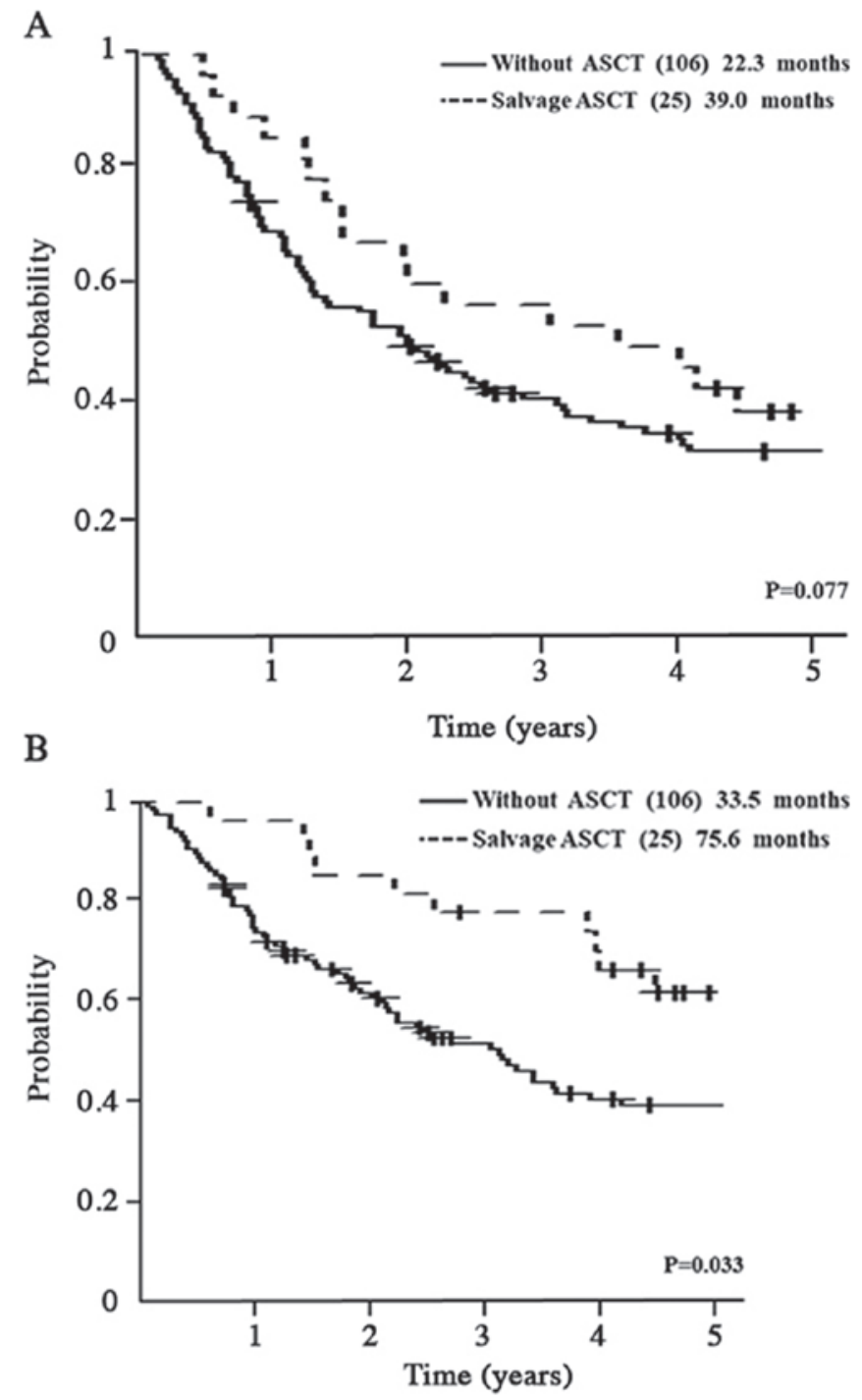

Figure 3. PFS and OS of patients with ASCT. (A) PFS of patients with ASCT. (B) OS of patients with ASCT. A total of 25 patients received ASCT. PFS, progression-free survival; OS, overall survival; ASCT, autologous stem cell transplantation. 


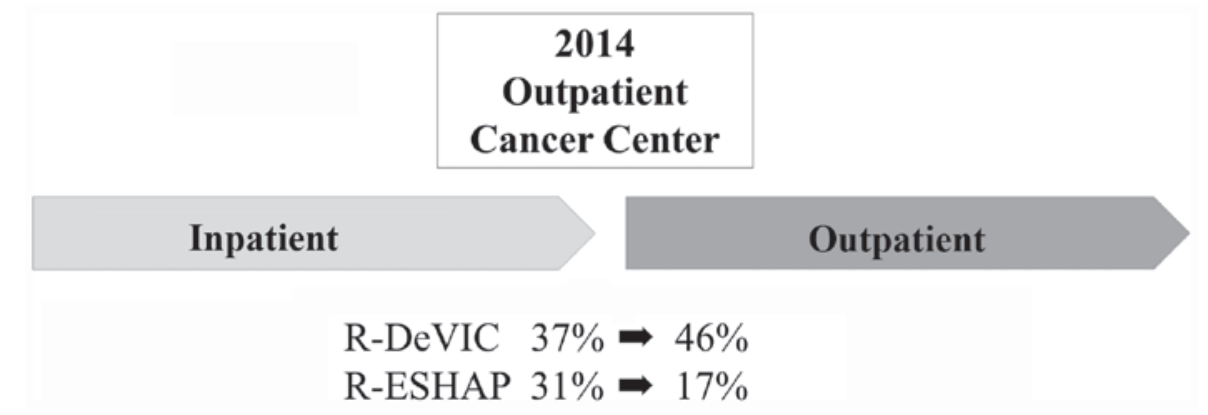

Figure 4. Transition of regimen in the outpatient chemotherapy unit. An outpatient chemotherapy unit was established in 2014. Prior to 2014, R-DeVIC was the most common regimen, followed by R-ESHAP. After 2014, R-DeVIC increased to 46\%, whereas R-ESHAP decreased to $17 \%$. R-DeVIC, rituximab, etoposide, dexamethasone, ifosfamide, carboplatin; R-ESHAP, rituximab, etoposide, solumedrol, cytarabine, cisplatin.

included R-CHASE (rituximab, cyclophosphamide, cytosine arabinoside, etoposide, dexamethasone) $(n=5)$, rituximab plus

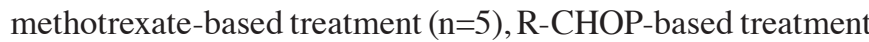
$(n=3)$, R-GDP (rituximab, gemcitabine, cisplatin, dexamethasone) ( $n=2)$, and R-EPOCH (rituximab, etoposide, vincristine, doxorubicin, cyclophosphamide, prednisolone) (n=1). Finally, $23 \%$ of patients underwent palliative therapy such as radiation, rituximab monotherapy, oral etoposide, or oral prednisolone.

Survival. Median OS by regimen was 45.7 (30.7-75.8) months for R-DeVIC, 41.8 (23.4-92.3) months for palliative therapy, 29.4 (14.1-98.1) months for R-ESHAP, and 28.5 (13.5-not applicable) months for aggressive regimens ( $\mathrm{P}=0.937$; Fig. 2).

Outcome of salvage ASCT. Twenty-five patients underwent ASCT, and had PFS2 of 39.0 (19.2-56.6) months compared with 22.3 (15.2-29.0) months in patients who did not undergo ASCT $(\mathrm{P}=0.077$; Fig. 3A). OS was 75.6 (51.8-not applicable) months in patients undergoing ASCT vs. 33.5 (25.6-45.6) months in patients who did not undergo ASCT ( $\mathrm{P}=0.033$; Fig. 3B).

Prognostic factors. Multivariate analysis was performed to identify risk factors associated with PFS2 and OS. Age over 70 years, male sex, PS $>3$, more than HI in IPI, and more than $\mathrm{HI}$ in NCCN-IPI were evaluated. NCCN-IPI [Hazard ratio (HR)]: 2.22, 95\% CI: 1.46-3.38, $\mathrm{P}=0.0001)$, male (HR: 1.60, 95\% CI: $1.06-2.41, \mathrm{P}=0.024)$, and $\mathrm{PS}>3$ (HR: $1.47,95 \% \mathrm{CI}$ : 1.06-2.03, $\mathrm{P}=0.022)$ remained as significant factors affecting PFS2 in multivariate analysis, whereas other factors were eliminated by backward stepwise selection. NCCN-IPI (HR: 2.56, 95\% CI: $1.56-4.20, \mathrm{P}=0.0002)$, IPI (HR: $1.68,95 \% \mathrm{CI}$ : 1.00-2.82, $\mathrm{P}=0.049$ ), and male (HR: $1.58,95 \% \mathrm{CI}: 1.00-2.47$, $\mathrm{P}=0.049$ ) remained as significant factors affecting survival.

Regimen transmission to outpatient chemotherapy. Our facilities established an outpatient chemotherapy unit in 2014, after which patients were primarily treated as outpatients. We analyzed the change in proportion of regimen prior to and after 2014 (Fig. 4). Before 2014, R-DeVIC was the most commonly administered regimen (37\%), followed by R-ESHAP (31\%). After 2014, R-DeVIC use increased to $46 \%$, whereas R-ESHAP decreased to $17 \%$.

Comparison between R-DeVIC and R-ESHAP. In our facilities, more than half of enrolled patients were treated with
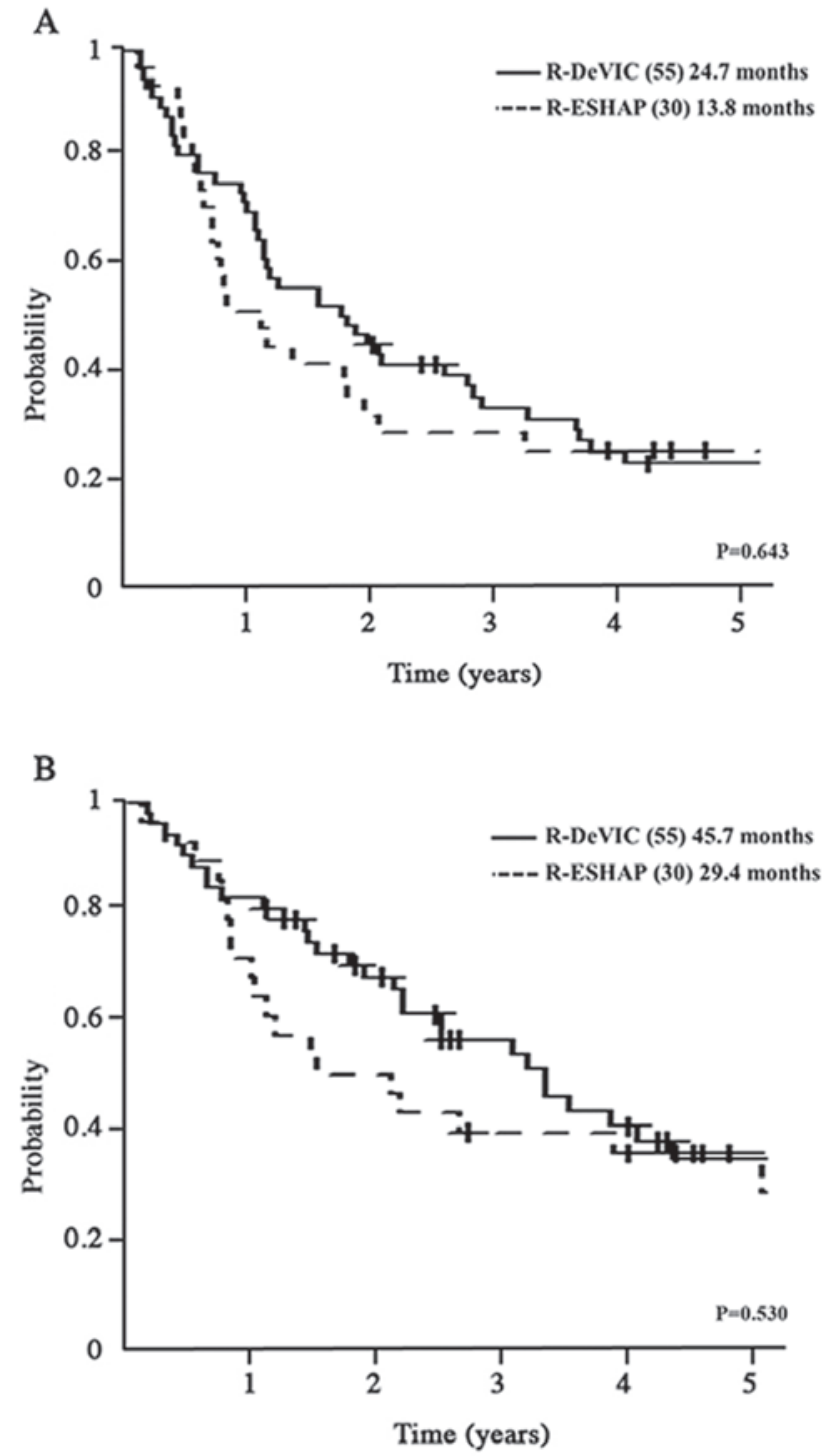

Figure 5. Comparison of PFS and OS between R-DeVIC and R-ESHAP. (A) Comparison of PFS between R-DeVIC and R-ESHAP. (B) Comparison of OS between R-DeVIC and R-ESHAP. PFS, progression-free survival; OS, overall survival; R-DeVIC, rituximab, etoposide, dexamethasone, ifosfamide, carboplatin; R-ESHAP, rituximab, etoposide, solumedrol, cytarabine, cisplatin.

either R-DeVIC or R-ESHAP as salvage therapy. Thus, we compared the efficacy of these two regimens. The treatment schedule is shown in Table II, and patient characteristics are 
Table III. Comparison between R-DeVIC and R-ESHAP.

\begin{tabular}{lcc}
\hline Characteristics & R-DeVIC & R-ESHAP \\
\hline Patients, $\mathrm{n}$ & 55 & 30 \\
Median age, years (range) & $70(35-84)$ & $61(41-68)$ \\
Male sex, $\%$ & 58 & 52 \\
PS $\geq 2$ at relapse, $\mathrm{n}(\%)$ & $2(4)$ & $6(19)$ \\
Stage at Dx, $\mathrm{n}(\%)$ & & \\
I & $6(11)$ & $1(3)$ \\
II & $8(15)$ & $4(13)$ \\
III & $13(24)$ & $7(23)$ \\
IV & $28(50)$ & $19(61)$ \\
Stage at relapse, $\mathrm{n}(\%)$ & & \\
I & $2(5)$ & $5(16)$ \\
II & $11(20)$ & $1(3)$ \\
III & $18(33)$ & $9(29)$ \\
IV & $24(42)$ & $16(52)$ \\
AE, $n$ & & \\
FN & 4 & 8 \\
Infection & 1 & 3 \\
Ileus & 1 & 1 \\
DIC & 0 & 1 \\
Heart disease & 0 & 1 \\
Early relapse, $\%$ & 44 & 77 \\
ASCT, $\%$ & 16 & 42 \\
\hline
\end{tabular}

R-DeVIC, rituximab, etoposide, dexamethasone, ifosfamide, carboplatin; R-ESHAP, rituximab, etoposide, solumedrol, cytarabine, cisplatin; Dx, diagnosis; AE, adverse event; FN, febrile neutropenia; DIC, disseminated intravascular coagulation; ASCT, autologous stem cell transplantation.

presented in Table III. In total, 55 patients were treated with R-DeVIC and 30 patients were treated with R-ESHAP. Median age was 70 years in the R-DeVIC group and 61 years in the R-ESHAP group. The most frequent adverse events other than hematological events (grade $\geq 3$ ) were febrile neutropenia $(n=4$ in R-DeVIC, $n=8$ in R-ESHAP) followed by infection $(n=1$ in R-DeVIC, $n=3$ in R-ESHAP). Early-relapse patients comprised $44 \%$ of the R-DeVIC group and $77 \%$ of the R-ESHAP group. PFS2 was 24.7 (15.5-39.0) months in the R-DeVIC group and 13.8 (10.2-27.4) months in the R-ESHAP group $(\mathrm{P}=0.643$; Fig. 5A), while OS was 45.7 (30.7-75.8) months in the R-DeVIC group and 29.4 (14.1-98.1) months in the R-ESHAP group ( $\mathrm{P}=0.53$; Fig. 5B).

\section{Discussion}

In the present analysis, R-DeVIC and R-ESHAP were used more frequently than other salvage regimens. Given that the choice of regimen was at the physician's discretion, it is difficult to determine the precise reason for selecting a particular regimen. However, we suggest that several factors may have contributed to regimen choice. First, R-ESHAP was administered to patients with more aggressive disease progression and worse condition, but with younger age. Physicians may have selected this regimen with the intention of subsequent salvage ASCT, as $42 \%$ of patients who received R-ESHAP later underwent ASCT. However, R-ESHAP treatment was associated with more AEs compared with R-DeVIC treatment. After 2014, the number of patients receiving R-ESHAP decreased with the establishment of our outpatient facility.

R-DeVIC was most commonly used regimen throughout the present study. The decisive factor in the selection of R-DeVIC vs. R-ESHAP is the need for hospitalization (Table II). As R-ESHAP is administered via a central venous catheter, patients require hospitalization. However, R-DeVIC can be given via a peripheral vein, and this type of regimen became more common after the establishment of the outpatient chemotherapy unit in 2014. Given that previous large trials have shown no difference between the salvage regimens, we suggest that the use of the outpatient unit is the likely reason for the increase in R-DeVIC use observed in our analysis.

Unfortunately, our findings failed to show a difference between R-DeVIC and R-ESHAP with respect to PFS2 and OS. However, patients that received R-DeVIC tended to have a better prognosis. As detailed above, R-ESHAP was selected in patients with more aggressive disease progression and worse condition. Thus, R-ESHAP may have been associated with a tendency toward worse prognosis.

ASCT has been previously shown to be an effective salvage therapy in patients with DLBCL $(4,5)$. In the present study, the number of patients who received ASCT was low compared with the patient population in SCHOLAR-1 study (6). This difference may be attributable to a higher median age in the present analysis (55 years vs. 68 years).

In multivariate analysis, NCCN-IPI was a significant predictive factor both in PFS2 and OS. NCCN-IPI has not been evaluated in second line treatment. NCCN-IPI might become a promising predictive scale for DLBCL.

The present study had some limitations, including its retrospective design, use of only two study sites, and small sample size. Furthermore, no genetic analysis using techniques such as fluorescence in situ hybridization (FISH) and immunostaining was performed. Genetic analysis is carried out in clinical settings, and its relevance to prognosis has been established. However, FISH is not covered by insurance in Japan, and there are disparities in immunostaining results between facilities. Thus, these evaluations are not necessarily performed routinely in clinical practice in Japan. We could not analyze QOL of patients although it is the important point to evaluate. The analyze of QOL should be conducted prospectively.

In conclusion, we were unable to identify the optimal salvage regimen for patients with DLBCL in the present study. However, we identified the effect of establishing an out-patient chemotherapy unit on salvage therapy selection, and anticipate that regimens administered via peripheral vein will become predominant in the future.

\section{Acknowledgements}

Not applicable.

\section{Funding}

No funding was received. 


\section{Availability of data and materials}

The datasets used and/or analyzed during the present study are available from the corresponding author on reasonable request.

\section{Authors' contributions}

$\mathrm{AN}$ and $\mathrm{SN}$ were responsible for drafting the article or revising it critically for important intellectual content. AN, SF, AS, TN, YA, YT, RS, AK, MH, HY, KI, TI and SN made substantial contributions to conception and design, and acquisition of data, and analysis and interpretation of data. All authors have approved the final version of this manuscript.

\section{Ethics approval and consent to participate}

The present retrospective study was approved by the Ethical Committee of Kansai Medical University (approval no. 2018063), and the requirement for written informed consent was waived.

\section{Patient consent for publication}

Not applicable.

\section{Competing interests}

The authors declare that they have no competing interests.

\section{References}

1. Friedberg JW: Relapsed/refractory diffuse large B-cell lymphoma. Hematology Am Soc Hematol Educ Program 2011: 498-505, 2011

2. Coiffier B, Lepage E, Briere J, Herbrecht R, Tilly H, Bouabdallah R, Morel P, Van Den, Salles G, Gaulard P, et al: CHOP chemotherapy plus rituximab compared with CHOP alone in elderly patients with diffuse large-B-cell lymphoma. N Engl J Med 346: 235-242, 2002.
3. Feugier P, Van Hoof, Sebban C, Solal-Celigny P, Bouabdallah R, Fermé C, Christian B, Lepage E, Tilly H, Morschhauser F, et al: Long-term results of the R-CHOP study in the treatment of elderly patients with diffuse large B-cell lymphoma: A study by the Groupe d'Etude des Lymphomes de l'Adulte. J Clin Oncol 23: 4117-4126, 2005.

4. Gisselbrecht C, Glass B, Mounier N, Singh Gill, Linch DC, Trneny M, Bosly A, Ketterer N, Shpilberg O, Hagberg H, et al: Salvage regimens with autologous transplantation for relapsed large B-cell lymphoma in the rituximab era. J Clin Oncol 28: 4184-4190, 2010

5. Crump M, Kuruvilla J, Couban S, MacDonald DA, Kukreti V, Kouroukis CT, Rubinger M, Buckstein R, Imrie KR, Federico M, et al: Randomized comparison of gemcitabine, dexamethasone, and cisplatin versus dexamethasone, cytarabine, and cisplatin chemotherapy before autologous stem-cell transplantation for relapsed and refractory aggressive lymphomas: NCIC-CTG LY.12. J Clin Oncol 32: 3490-3496, 2014.

6. Crump M, Neelapu SS, Farooq U, Van Den, Kuruvilla J, Westin J, Link BK, Hay A, Cerhan JR, Zhu L, et al: Outcomes in refractory diffuse large B-cell lymphoma: Results from the international SCHOLAR-1 study. Blood 130: 1800-1808, 2017.

7. Ziepert M, Hasenclever D, Kuhnt E, Glass B, Schmitz N, Pfreundschuh $\mathrm{M}$ and Loeffler M: Standard International prognostic index remains a valid predictor of outcome for patients with aggressive CD20+B-cell lymphoma in the rituximab era. J Clin Oncol 28: 2373-2380, 2010

8. Zhou Z, Sehn LH, Rademaker AW, Gordon LI, Lacasce AS Crosby-Thompson A, Vanderplas A, Zelenetz AD, Abel GA, Rodriguez MA, et al: An enhanced International Prognostic Index (NCCN-IPI) for patients with diffuse large B-cell lymphoma treated in the rituximab era. Blood 123: 837-842, 2014.

9. Kanda Y: Investigation of the freely available easy-to-use software 'EZR' for medical statistics. Bone Marrow Transplant 48: 452-458, 2013 